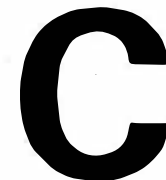
'est par la génétique moléculaire que les Sciences du vivant ont vu émerger leur première grande théorie cohérente. Au cours des vingt dernières années, le codage de l'information génétique et les mécanismes de régulation de son expression ont représenté la seule véritable axiomatique de la biologie, applicable aussi bien à toutes les espèces qu'à toutes les fonctions. Mais on assiste actuellement à l'émergence d'une nouvelle théorie générale au niveau de notre représentation des régulations physiologiques : la théorie de la communication cellulaire, qui, comme la génétique moléculaire, ne connaît pas de frontières d'espèce ou de fonction.

Cette théorie s'appuie sur trois constatations : les signaux qui règlent l'activité de chaque cellule sont très nombreux; ils sont banalisés, au sens où les mêmes signaux se trouvent associés à toutes les fonctions et interviennent au niveau de tous les organes ; enfin, les mécanismes de reconnaissance sont universels. La plupart des cellules de l'organisme sont capables de reconnaître des dizaines de signaux différents - hormones, neuromédiateurs, médiateurs de l'immunité. Par exemple, les neurones du système nerveux central reçoivent en moyenne 1000 afférences et envoient autant de projections qui vont former synapse sur d'autres cellules. Dans ces conditions, le problème de la communication cellulaire consiste essentiellement à extraire des messages d'un extraordinaire bruit de fond. Ces messages ne peuvent être assimilés à la simple somme des effets de chaque signal ; les signaux peuvent en effet se renforcer ou s'atténuer les uns les autres. L'information intercellulaire est donc elle-même codée : la spécificité et la sélectivité des messages dépendent de la combinaison des signaux élémentaires reçus par une cellule cible, ou éventuellement de l'ordre de présentation de ces signaux (et donc, pas seulement de la structure chimique des ligands).

\section{Claude Kordon}

Directeur de recherche au Cnrs Directeur de l'unité U.159 de l'Inserm

\section{ADRESSE}

C. Kordon : Inserm U.159, centre Paul-Broca, 2 ter, rue d'Alesia, 75014 Paris.

$m / s n^{\circ} 3$ vol. 3 , mars 87 
La deuxième observation concerne la banalisation des signaux. Il y a une vingtaine d'années, on ne suspectait l'existence que d'une dizaine de neuromédiateurs. On en connaît aujourd'hui plus de 60 ; une bonne partie des signaux nouvellement reconnus n'a pas été découverte dans le système nerveux central, mais dans des tissus périphériques, en particulier le tractus gastro-intestinal. La distinction hormone/médiateur n'a plus guère de sens : les mêmes substances (des neuropeptides comme la vasopressine, l'angiotensine, la bêta-endorphine, ou des médiateurs aminergiques comme la noradrénaline et la dopamine) sont à la fois médiateurs dans le système nerveux central et hormones à la périphérie. L'étendue de leurs effets tient essentiellement aux conditions de leur diffusion et de leur transport : régulations synaptiques et paracrines impliquent des signaux à diffusion locale, tandis que les régulations endocrines font appel à des signaux transportés sur de plus longues distances.

Enfin, les mécanismes d'émission et de réception des signaux sont universels. Quels que soient l'espèce ou le tissu considérés, un récepteur se résume au fond à deux éléments essentiels : un déterminant extramembranaire de la reconnaissance, et un effecteur intracellulaire. L'effecteur intracellulaire est toujours, soit un canal ionique transmembranaire, soit une protéine kinase. Dans les cas les plus simples, séquence de reconnaissance et activité enzymatique peuvent être portées par une même molécule (cas de certains oncogènes) ; mais reconnaissance et transduction sont généralement associées au sein d'entités polyprotéiques (par exemple, dans le récepteur nicotinique ou le récepteur de l'insuline, des sous-unités différentes expriment la reconnais-

$m / s n^{\circ} 3$ vol. 3 , mars 87 sance et l'activité du canal ionique ou de la protéine kinase). Enfin, certains récepteurs comportent un niveau d'amplification supplémentaire : dans ce cas, canal ou protéine kinase sont mis en jeu par des seconds messagers, produits par des enzymes (phospholipases ou adénylate cyclase), elles-mêmes couplées au récepteur par un intermédiaire de couplage (la famille des protéines $G$ ).

L'universalité des processus de réception se manifeste également par le fait qu'un récepteur déterminé est toujours couplé de la même manière : un récepteur $\mathrm{X}^{\mathrm{y}}$ (par exemple, le récepteur D1 de la dopamine, ou le récepteur $\beta$ adrénergique) présente invariablement le même mode de couplage (en l'occurrence à une protéine kinase par l'intermédiaire de l'adénylate cyclase), quels que soient l'espèce ou le tissu considérés.

La banalisation des mécanismes de signalisation cellulaire va de pair avec leur diversification. Les séquences nucléotidiques codant pour la partie active d'un signal intercellulaire peuvent être répétées plusieurs fois sur un même gène (comme le pentapeptide metenképhaline sur le gène de la proenképhaline) ou sur des gènes différents (famille des 3 gènes opiacés). Des altérations de la séquence nucléotidique peuvent également entraîner la substitution d'un ou plusieurs acides aminés (cas de la met-enképhaline et de la leu-enképhaline, ou des substances $P$ et $K$ codées par le gène des tachykinìnes). Parallèlement, se produit une diversification correspondante des récepteurs. Les règles en sont encore mal connues. Signalons cependant que l'émetteur et le récepteur du signal peuvent exercer l'un sur l'autre une influence réciproque : les greffes de neurones peuvent ainsi exprimer des médiateurs dif- férents selon la nature des cellules avec lesquelles elles établissent des contacts synaptiques.

Enfin, la spécificité de la réponse cellulaire peut s'exprimer à travers les mécanismes de couplage euxmêmes. Bien que chaque récepteur soit couplé de manière constante, les chaînes de couplage de plusieurs récepteurs peuvent interagir entre elles de manière spécifique à chaque type cellulaire. Les cellules de même type présentent donc une "grille de lecture " (le " programme latent " de transduction) qui leur est propre et leur permet de décrypter un même message autrement que ne le ferait un autre type cellulaire. Le répertoire des ligands et des récepteurs s'est évidemment beaucoup enrichi au cours de l'évolution. Cet enrichissement pourrait bien être le fruit d'une stratégie active de diversification du génome, analogue aux réarrangements géniques mis en évidence par les immunologistes au cours de la réponse lymphocytaire à un antigène inconnu (mais évidemment à une toute autre échelle de temps !). Sous l'effet combiné de cette stratégie d'exploration de la diversité et de la pression de sélection, des mécanismes de complexité croissante ont pu se développer à partir de processus élémentaires.

Le présent numéro donne quelques exemples de tels mécanismes. Pris dans leur ensemble, ils s'inscrivent bien dans la nouvelle représentation des systèmes de signalisation cellulaire. Celle-ci peut sans doute paraître un peu abstraite : elle n'en est que plus facile à généraliser à toutes les fonctions de régulation. C'est ainsi que l'analyse des " codes " de la communication cellulaire tendra de plus en plus à se substituer au langage traditionnel de la physiologie et de la pathologie des fonctions 